\title{
Explicit upper bound for the average number of divisors of irreducible quadratic polynomials
}

\author{
Kostadinka Lapkova $^{1}$ (D)
}

Received: 6 December 2016 / Accepted: 19 May 2017 / Published online: 27 May 2017

(C) The Author(s) 2017. This article is an open access publication

\begin{abstract}
Consider the divisor sum $\sum_{n \leq N} \tau\left(n^{2}+2 b n+c\right)$ for integers $b$ and $c$. We extract an asymptotic formula for the average divisor sum in a convenient form, and provide an explicit upper bound for this sum with the correct main term. As an application we give an improvement of the maximal possible number of $D(-1)$ quadruples.
\end{abstract}

Keywords Number of divisors · Quadratic polynomial · Dirichlet convolution

Mathematics Subject Classification Primary 11N56; Secondary 11D09

\section{Introduction}

Let $\tau(n)$ denote the number of positive divisors of the integer $n$ and $P(x) \in \mathbb{Z}[x]$ be a polynomial. Due to their numerous applications average sums of divisors

$$
\sum_{n=1}^{N} \tau(P(n))
$$

have obtained a lot of attention, e.g. in [4,7-9,20,21]. The current paper aims to improve the author's results from [11] concerning explicit upper bound for divisor

Communicated by A. Constantin.

$凶$ Kostadinka Lapkova

lapkova@math.tugraz.at

1 Institute of Analysis and Number Theory, Graz University of Technology, Kopernikusgasse 24/II, 8010 Graz, Austria 
sums over certain irreducible quadratic polynomials $P(x)$. More precisely, while the upper bound obtained in [11] appears to be the first one of the right order of magnitude $N \log N$ with completely explicit constants, it does not provide the expected constant in the main term. Here we fill this gap achieving the correct main term as in the asymptotic formula for the corresponding divisor sum. A more detailed introduction to the subject can be found in $[11,15]$.

Only very recently [5,12] provided asymptotic formulae for the sum (1.1) for some reducible quadratic polynomials, whereas the asymptotic formulae for irreducible quadratic polynomials are classical, due to Scourfield [20], Hooley [10] and McKee [13-15].

Let $P(x)=x^{2}+B x+C$ for integers $B$ and $C$ such that $P(x)$ is irreducible. Denote

$$
\rho(d, P)=\rho(d):=\#\{0 \leq m<d: P(m) \equiv 0 \quad(\bmod d)\} .
$$

Then we have

$$
\sum_{n \leq N} \tau(P(n)) \sim \lambda N \log N
$$

as $N \rightarrow \infty$, for some $\lambda$ depending on $B$ and $C$. Hooley [10] showed that for irreducible $P(x)=x^{2}+C$ we have

$$
\lambda=\frac{8}{\pi^{2}} \sum_{\alpha=0}^{\infty} \frac{\rho\left(2^{\alpha}\right)}{2^{\alpha}} \sum_{\substack{d^{2} \mid C \\ d \text { odd }}} \frac{1}{d} \sum_{\substack{l=1 \\ l \text { odd }}}^{\infty} \frac{1}{l}\left(\frac{-C / d^{2}}{l}\right),
$$

while for the more general $P(x)=x^{2}+B x+C$ with $\Delta=B^{2}-4 C$ not a square, McKee [15] obtained

$$
\lambda= \begin{cases}12 H^{*}(\Delta) /(\pi \sqrt{|\Delta|}) & \text { if } \Delta<0, \\ 12 H^{*}(\Delta) \log \epsilon_{\Delta} /\left(\pi^{2} \sqrt{\Delta}\right) & \text { if } \Delta>0,\end{cases}
$$

where $H^{*}(\Delta)$ is a weighted class number, and $\epsilon_{\Delta}$ for $\Delta>0$ is a fundamental unit. Nevertheless we would prove independently the asymptotic formula for the specialized irreducible quadratic polynomials in which we are interested. We achieve a compact main term with a constant $\lambda$ which is easier to formulate and easy to compare with the main term in our explicit upper bound.

Our first result is the following theorem.

Theorem 1 Let $b$ and $c$ be integers, such that the discriminant $\delta:=b^{2}-c$ is nonzero and square-free, and $\delta \not \equiv 1(\bmod 4)$. Then for $N \rightarrow \infty$ we have the asymptotic formula

$$
\sum_{n=1}^{N} \tau\left(n^{2}+2 b n+c\right)=\frac{2}{\zeta(2)} L(1, \chi) N \log N+\mathcal{O}(N)
$$


where $\chi(n)=\left(\frac{4 \delta}{n}\right)$ for the Kronecker symbol $(\dot{*})$.

In particular, when we consider the polynomial $f(n)=n^{2}+1$ we have $\delta=-1$ and $\chi(n)=\left(\frac{-4}{n}\right)$ is the non-principal primitive Dirichlet character modulo 4 . Then $L(1, \chi)=\sum_{n=0}^{\infty}(-1)^{n} /(2 n+1)=\pi / 4$ by the formula of Leibniz. We substitute $\zeta(2)=\pi^{2} / 6$ in Theorem 1 and recover the well-known asymptotic

$$
\sum_{n=1}^{N} \tau\left(n^{2}+1\right)=\frac{3}{\pi} N \log N+\mathcal{O}(N)
$$

We note that $4 \delta=\Delta$ is a fundamental discriminant and the weighted class number $H^{*}(\Delta)$ in McKee's formula (1.3) is the usual class number for $\Delta>0$ and $\Delta<-4$. When $\delta=-1$ we have an adjustment by the corresponding root number. Indeed, for negative discriminants $H^{*}(\Delta)$ is the Hurwitz class number for which the precise relation with the usual class number is described for example in ([3], Lemma 5.3.7). Then the Dirichlet class number formula yields the equality of the constant $2 L(1, \chi) / \zeta(2)$ with $\lambda$ from (1.3) for the polynomials we consider in Theorem 1. Our proof, however, is different than McKee's [13-15], and is rather similar to Hooley's argument from [10].

The novelty in this paper is the following explicit upper bound, which achieves the correct main term as in the asymptotic formula from Theorem 1.

Theorem 2 Let $f(n)=n^{2}+2 b n+c$ for integers $b$ and $c$, such that the discriminant $\delta:=b^{2}-c$ is non-zero and square-free, and $\delta \not \equiv 1(\bmod 4)$. Assume also that for $n \geq 1$ the function $f(n)$ is non-negative. Then for any $N \geq 1$ satisfying $f(N) \geq f(1)$, and $X:=\sqrt{f(N)}$, we have the inequality

$$
\begin{aligned}
\sum_{n=1}^{N} \tau\left(n^{2}+2 b n+c\right) \leq & \frac{2}{\zeta(2)} L(1, \chi) N \log X \\
& +\left(2.332 L(1, \chi)+\frac{4 M_{\delta}}{\zeta(2)}\right) N+\frac{2 M_{\delta}}{\zeta(2)} X \\
& +8 M_{\delta} \frac{N}{\sqrt{X}}+4 M_{\delta} \sqrt{X} \\
& +8 M_{\delta} \frac{N}{X}+4 M_{\delta},
\end{aligned}
$$

where $\chi(n)=\left(\frac{4 \delta}{n}\right)$ for the Kronecker symbol $(\dot{*})$ and

$$
M_{\delta}= \begin{cases}\frac{4}{\pi^{2}} \delta^{1 / 2} \log 4 \delta+\frac{8}{\pi^{2}} \delta^{1 / 2} \log \log 4 \delta+3 \delta^{1 / 2}, & \text { if } \delta>0 \\ \frac{1}{\pi}|\delta|^{1 / 2} \log 4|\delta|+\frac{2}{\pi}|\delta|^{1 / 2} \log \log 4|\delta|+2|\delta|^{1 / 2}, & \text { if } \delta<0\end{cases}
$$


Note that we can formulate the theorem by extracting positive constants $C_{1}, C_{2}, C_{3}$ such that

$$
\sum_{n=1}^{N} \tau\left(n^{2}+2 b n+c\right) \leq \frac{2}{\zeta(2)} L(1, \chi) N \log N+C_{1} N+C_{2} \sqrt{N}+C_{3}
$$

because $X=\sqrt{f(N)}=N+\mathcal{O}(1)$. However we restrained ourselves from doing so in order to keep our result more precise for eventual numerical applications. Still, improvements of the lower order terms are very likely, for example by applying the theory developed by Akhilesh and Ramaré [1].

When we know the exact form of the quadratic polynomial and the corresponding character, we might achieve even better upper bounds. This is the case of the polynomial $f(n)=n^{2}+1$ when the following corollary holds.

Corollary 3 For any integer $N \geq 1$ we have

$$
\sum_{n \leq N} \tau\left(n^{2}+1\right)<\frac{3}{\pi} N \log N+4.264 N+8 \sqrt{N}+8 .
$$

Just as in [11] we give an application of the latter inequality. Define a $D(n)-m$ tuple for a nonzero integer $n$ and a positive integer $m$ to be a set of $m$ integers such that the product of any two of them increased by $n$ is a perfect square. It is conjectured that there are no $D(-1)$-quadruples but currently it is only known that there are at most $4.7 \cdot 10^{58} D(-1)$-quadruples [11]. The latter number upgraded the previous maximal possible bound $3.01 \times 10^{60}$ due to Trudgian [19], which in turn improved results from $[2,6]$.

Plugging the upper bound of Corollary 3 in the proof of ([6], Theorem 1.3) from the paper of Elsholtz, Filipin and Fujita we obtain another slight improvement.

Corollary 4 There are at most $3.713 \cdot 10^{58} \mathrm{D}(-1)$-quadruples.

\section{Proof of Theorem 2}

We start with proving the explicit upper bound in Theorem 2, as the claims required for the proof of Theorem 1 can be easily adapted by the lemmae from this section.

Let us consider the polynomial $f(n)=n^{2}+2 b n+c$ with integer coefficients $b$ and $c$, and the function $\rho(d)$ defined in (1.2) counts the roots of $f(n)$ in a full residue system modulo $d$. Let $\delta=b^{2}-c$ and $\chi(n)=\left(\frac{4 \delta}{n}\right)$ for the Kronecker symbol $(\therefore)$.

The core of the proofs of both Theorems 1 and 2 is the following convolution lemma which we proved in [11].

Lemma 1 ([11], Lemma 2.1) Let $\delta=b^{2}-c$ be square-free and $\delta \not \equiv 1(\bmod 4)$. Then we have the identity

$$
\rho(d)=\sum_{l m=d} \mu^{2}(l) \chi(m)
$$


The proof of Lemma 1 is based on elementary facts about the function $\rho(d)$ at prime powers and manipulations of the Dirichlet series it generates.

Further we need the following explicit estimates.

Lemma 2 For any integer $N \geq 1$ we have

$$
\sum_{n \leq N} \mu^{2}(n)=\frac{N}{\zeta(2)}+E_{1}(N)
$$

where $\left|E_{1}(N)\right| \leq 2 \sqrt{N}+2$.

Proof Let us denote $Q(N):=\sum_{n \leq N} \mu^{2}(n)$ for $N \geq 1$. Then we can write

$$
Q(N)=\sum_{n \leq N} \sum_{d^{2} \mid n} \mu(d)=\sum_{1 \leq d \leq \sqrt{N}} \mu(d) \sum_{\substack{1 \leq n \leq N \\ d^{2} \mid n}} 1=\sum_{1 \leq d \leq \sqrt{N}} \mu(d)\left[\frac{N}{d^{2}}\right]
$$

Using $\sum_{n=1}^{\infty} \mu(d) / d^{2}=1 / \zeta(2)$ we see that

$$
E_{1}(N)=Q(N)-\frac{N}{\zeta(2)}=-\sum_{1 \leq d \leq \sqrt{N}} \mu(d)\left\{\frac{N}{d^{2}}\right\}-N \sum_{d>\sqrt{N}} \frac{\mu(d)}{d^{2}}
$$

hence

$$
\left|E_{1}(N)\right| \leq \sqrt{N}+N\left|\sum_{d>\sqrt{N}} \frac{\mu(d)}{d^{2}}\right|
$$

On its turn for every real $x>1$

$$
\left|\sum_{d>x} \frac{\mu(d)}{d^{2}}\right| \leq \sum_{d>x} \frac{1}{d^{2}}<\int_{x-1}^{\infty} \frac{d t}{t^{2}}=\frac{1}{x-1}
$$

and $N /(\sqrt{N}-1) \leq \sqrt{N}+2$ for $N \geq 4$. Now from (2.1) it follows that $Q(N)=$ $N / \zeta(2)+E_{1}(N)$ and $\left|E_{1}(N)\right| \leq 2(\sqrt{N}+1)$ for $N \geq 4$. It is trivial to check that the inequality

$$
\left|Q(N)-\frac{N}{\zeta(2)}\right| \leq 2 \sqrt{N}+2
$$

holds also for $N \in\{1,2,3\}$ and this proves the statement of the lemma.

The following effective Pólya-Vinogradov inequality is due to Pomerance. 
Lemma 3 ([16], Theorem 1) Let

$$
M_{\chi}:=\max _{L, P}\left|\sum_{n=L}^{P} \chi(n)\right|
$$

for a primitive character $\chi$ to the modulus $q>1$. Then

$$
M_{\chi} \leq \begin{cases}\frac{2}{\pi^{2}} q^{1 / 2} \log q+\frac{4}{\pi^{2}} q^{1 / 2} \log \log q+\frac{3}{2} q^{1 / 2}, & \chi \text { even } \\ \frac{1}{2 \pi} q^{1 / 2} \log q+\frac{1}{\pi} q^{1 / 2} \log \log q+q^{1 / 2}, & \chi \text { odd } .\end{cases}
$$

The next lemma is critical for obtaining the right main term in the explicit upper bound of Theorem 2 .

Lemma 4 For any integer $N \geq 1$ we have

$$
\sum_{n \leq N} \frac{\chi(n)}{n}=L(1, \chi)+E_{2}(N)
$$

where $\left|E_{2}(N)\right| \leq 2 M_{\delta} / N$ and $M_{\delta}$ is the constant defined in Theorem 2.

Proof This statement, especially the estimate of the error term, is much less trivial and follows from the effective Pólya-Vinogradov inequality of Pomerance. First we notice that

$$
\sum_{n \leq N} \frac{\chi(n)}{n}=\sum_{n=1}^{\infty} \frac{\chi(n)}{n}-\sum_{n>N} \frac{\chi(n)}{n}=L(1, \chi)+E_{2}(N) .
$$

Let us denote $X(N):=\sum_{1 \leq n \leq N} \chi(n)$ for any positive integer $N$. Then by Abel's summation it follows that for any positive integer $Z>N$ we have

$$
\begin{aligned}
\left|\sum_{N<n \leq Z} \frac{\chi(n)}{n}\right| & =\left|\frac{X(Z)}{Z}-\frac{X(N)}{N}+\int_{N}^{Z} \frac{X(t)}{t^{2}} d t\right| \\
& \leq \frac{|X(Z)|}{Z}+\frac{|X(N)|}{N}+\int_{N}^{Z} \frac{|X(t)|}{t^{2}} d t .
\end{aligned}
$$

Recall the definition of the quantity $M_{\chi}$ in Lemma 3. Then

$$
\left|\sum_{N<n \leq Z} \frac{\chi(n)}{n}\right| \leq M_{\chi}\left(\frac{1}{Z}+\frac{1}{N}+\int_{N}^{Z} \frac{d t}{t^{2}}\right)=\frac{2 M_{\chi}}{N},
$$

which is uniform in $Z$. Therefore $\left|E_{2}(N)\right|=\left|\sum_{n>N} \chi(n) / n\right| \leq 2 M_{\chi} / N$. Now by the conditions on $\delta$ to be square-free and not congruent to 1 modulo 4 it follows that 
the discriminant $4 \delta$ is fundamental, thus the character $\chi(n)=\left(\frac{4 \delta}{n}\right)$ is primitive with a conductor $4|\delta|$. Then according to Lemma $3 M_{\chi} \leq M_{\delta}$ and this proves the statement.

The following lemma is due to Ramaré. One could easily extract the right main term from Lemma 2 through Abel summation but the estimate of the minor term requires computer calculations.

Lemma 5 ([17], Lemma 3.4) Let $x \geq 1$ be a real number. We have

$$
\sum_{n \leq x} \frac{\mu^{2}(n)}{n} \leq \frac{\log x}{\zeta(2)}+1.166
$$

Let us start with the proof of Theorem 2. Note that from the condition $f(N) \geq f(1)$ and the convexity of the function $f(x)$ we have $f(N)=\max _{1 \leq n \leq N} f(n)$. Using the Dirichlet hyperbola method we have

$$
\begin{aligned}
\sum_{1 \leq n \leq N} \tau(f(n)) & =\sum_{1 \leq n \leq N} \sum_{d \mid f(n)} 1 \leq 2 \sum_{1 \leq n \leq N} \sum_{\substack{d \leq \sqrt{f(n)} \\
d \mid f(n)}} 1 \\
& =2 \sum_{1 \leq d \leq \sqrt{f(N)}} \sum_{\substack{1 \leq n \leq N \\
d+\mathcal{O}(1) \leq n}} \sum_{d \mid f(n)} 1 \leq 2 \sum_{\substack { 1 \leq d \leq \sqrt{f(N)} \\
\begin{subarray}{c}{1 \leq n \leq N \\
d \mid f(n){ 1 \leq d \leq \sqrt { f ( N ) } \\
\begin{subarray} { c } { 1 \leq n \leq N \\
d | f ( n ) } }\end{subarray}} 1 .
\end{aligned}
$$

Recall the definition (1.2) of the function $\rho(d)$. Then the innermost sum equals $[N / d]$ copies of $\rho(d)$ plus a remaining part smaller than $\rho(d)$. Recall that $X=\sqrt{f(N)}$. Then

$$
\sum_{1 \leq n \leq N} \tau(f(n)) \leq 2 \sum_{1 \leq d \leq X}\left(\frac{N}{d} \rho(d)+\rho(d)\right)=2 N \sum_{1 \leq d \leq X} \frac{\rho(d)}{d}+2 \sum_{1 \leq d \leq X} \rho(d) .
$$

From the convolution identity stated in Lemma 1 it immediately follows that

$$
\sum_{d \leq X} \rho(d)=\sum_{l m \leq X} \mu^{2}(l) \chi(m)=\sum_{l \leq X} \mu^{2}(l) \sum_{m \leq X / l} \chi(m) .
$$

Recall the definition of $M_{\chi}$ and the Pomerance bounds $M_{\chi} \leq M_{\delta}$. Then using also Lemma 2 we get

$$
\sum_{d \leq X} \rho(d) \leq M_{\delta} \sum_{l \leq X} \mu^{2}(l) \leq M_{\delta}\left(\frac{X}{\zeta(2)}+2 \sqrt{X}+2\right)
$$

Similarly by the convolution property of the function $\rho(d)$ and Lemma 4 we can write

$$
\sum_{d \leq X} \frac{\rho(d)}{d}=\sum_{l \leq X} \frac{\mu^{2}(l)}{l} \sum_{m \leq X / l} \frac{\chi(m)}{m} \leq \sum_{l \leq X} \frac{\mu^{2}(l)}{l}\left(L(1, \chi)+2 M_{\delta} \frac{l}{X}\right)
$$


One could go further using Lemma 5 and again Lemma 2. We obtain

$$
\begin{aligned}
\sum_{d \leq X} \frac{\rho(d)}{d} & \leq L(1, \chi) \sum_{l \leq X} \frac{\mu^{2}(l)}{l}+\frac{2 M_{\delta}}{X} \sum_{l \leq X} \mu^{2}(l) \\
& \leq L(1, \chi)\left(\frac{\log X}{\zeta(2)}+1.166\right)+\frac{2 M_{\delta}}{X}\left(\frac{X}{\zeta(2)}+2 \sqrt{X}+2\right)
\end{aligned}
$$

Plugging the estimates (2.3) and (2.5) in (2.2) gives the statement of Theorem 2.

Let us prove Corollary 3. For the polynomial $f(n)=n^{2}+1$ we can be more precise as the condition $d \leq \sqrt{f(n)}$ is equivalent to $d \leq n$. Then after applying more carefully the hyperbola method and changing the order of summation we obtain

$$
\begin{aligned}
\sum_{n \leq N} \tau\left(n^{2}+1\right) & \left.=2 \sum_{n \leq N} \sum_{\substack{d \leq n \\
d \mid n^{2}+1}} 1=2 \sum_{\substack{1 \leq d \leq N \\
d \leq n \leq N \\
d \mid n^{2}+1}} \sum_{\substack { 1 \leq d \leq N \\
\begin{subarray}{c}{1 \leq n \leq N \\
d \mid n^{2}+1{ 1 \leq d \leq N \\
\begin{subarray} { c } { 1 \leq n \leq N \\
d | n ^ { 2 } + 1 } }\end{subarray}} 1-\sum_{\substack{1 \leq n<d \\
d \mid n^{2}+1}} 1\right) \leq 2 N \sum_{1 \leq d \leq N} \frac{\rho(d)}{d} \\
& =2 \sum_{1 \leq N}\left(\sum_{1 \leq 1}\right.
\end{aligned}
$$

Similarly to (2.4) we have

$$
\sum_{1 \leq d \leq N} \frac{\rho(d)}{d}=\sum_{l \leq N} \frac{\mu^{2}(l)}{l} \sum_{m \leq N / l} \frac{\chi(m)}{m} \leq \sum_{l \leq N} \frac{\mu^{2}(l)}{l}\left(L(1, \chi)+2 M_{\chi} \frac{l}{N}\right) .
$$

In this case $M_{\chi} \leq 1$ and $L(1, \chi)=\pi / 4$, and application of Lemmas 5 and 2 gives

$$
\begin{aligned}
\sum_{1 \leq d \leq N} \frac{\rho(d)}{d} & \leq \frac{\pi}{4} \sum_{l \leq N} \frac{\mu^{2}(l)}{l}+\frac{2}{N} \sum_{l \leq N} \mu^{2}(l) \\
& \leq \frac{\pi}{4}\left(\frac{\log N}{\zeta(2)}+1.166\right)+\frac{2}{N}\left(\frac{N}{\zeta(2)}+2 \sqrt{N}+2\right)
\end{aligned}
$$

After numerical approximation of the constants we conclude the validity of Corollary 3.

\section{Proof of Theorem 1}

Let again $f(n)=n^{2}+2 b n+c$. We can assume that $f(n)$ is non-negative for $n \geq 1$ as for $N$ large enough $f(N)=\max _{1 \leq n \leq N} f(n)$ and the contribution of the finitely many integers $n$ for which $f(n)<0$ will be negligible compared to the main term. 
As in the proof of Theorem 2 and using (2.2) and (2.3) it is easy to see that

$$
\begin{aligned}
\sum_{n \leq N} \tau(f(n)) & =2 N \sum_{d \leq N} \frac{\rho(d)}{d}+\mathcal{O}\left(\sum_{d \leq N} \rho(d)\right)+\mathcal{O}(N) \\
& =2 N \sum_{d \leq N} \frac{\rho(d)}{d}+\mathcal{O}(N)
\end{aligned}
$$

We need the following estimates.

Lemma 6 For $N \rightarrow \infty$ we have the asymptotic formulae

$$
\sum_{n \leq N} \frac{\mu^{2}(n)}{n}=\frac{\log N}{\zeta(2)}+\mathcal{O}(1)
$$

and

$$
\sum_{n \leq N} \frac{\chi(n)}{n}=L(1, \chi)+\mathcal{O}(1 / N)
$$

Proof The first asymptotic formula follows easily after Abel transformation of the classical formula

$$
\sum_{n \leq N} \mu^{2}(n)=\frac{N}{\zeta(2)}+\mathcal{O}(\sqrt{N})
$$

which in its turn obviously follows from the explicit version in Lemma 2. The second statement can be deduced from Lemma 4.

Then the asymptotic estimate corresponding to (2.4) is

$$
\begin{aligned}
\sum_{d \leq N} \frac{\rho(d)}{d}= & \sum_{l \leq N} \frac{\mu^{2}(l)}{l}(L(1, \chi)+\mathcal{O}(l / N))=L(1, \chi) \sum_{l \leq N} \frac{\mu^{2}(l)}{l} \\
& +\mathcal{O}\left(\frac{1}{N} \sum_{l \leq N} \mu^{2}(l)\right)=L(1, \chi)\left(\frac{\log N}{\zeta(2)}+\mathcal{O}(1)\right)+\mathcal{O}(1) \\
= & \frac{L(1, \chi)}{\zeta(2)} \log N+\mathcal{O}(1)
\end{aligned}
$$

Now plugging (3.2) into (3.1) gives Theorem 1. 


\section{Some examples}

In our preceding paper [11] we gave several examples of polynomials for which Theorem 2 can provide effective upper bound of the average divisor sum. There we compared the coefficient of the main term in the asymptotic formula of McKee with the one from our upper bound. The current paper achieves equality of the two coefficients but there is one more computation to be made, so that our result could be useful numerically, this of the special value of the Dirichlet $L$-function $L(s, \chi)$ at $s=1$.

Formulae for computing $L(1, \chi)$ in the two separate cases $\delta<0$ and $\delta>0$ are given in ([22], Theorem 4.9). When $\delta<0$ this is implemented by the SAGE function quadratic_L_function_exact $(1,4 \delta)$ whose values are computed for several examples in the table below. When $\delta>0$ one can find the approximate value of $L(1, \chi)$ by finding first the corresponding Dirichlet character (of modulus $4 \delta$, order 2 , even and primitive) in the Refine search platform from LMFDB (The $L$-functions and modular forms database [18]).

The following table lists some examples.

\begin{tabular}{llll}
\hline$f(n)$ & $\delta$ & $\chi$ & $L(1, \chi)$ \\
\hline$n^{2}+1$ & -1 & $\left(\frac{-1}{\cdot}\right)$ & $\pi / 4-0.7854$ \\
$n^{2}+10 n+27$ & -2 & $\left(\frac{-8}{\cdot}\right)$ & $\sqrt{2} / 4 \pi-1.1108$ \\
$n^{2}+4 n+10$ & -6 & $\left(\frac{-24}{\cdot}\right)$ & $\sqrt{6} / 6 \pi-1.2826$ \\
$n^{2}+10 n-10$ & 35 & $\left(\frac{140}{\cdot}\right)$ & $\sim 0.8377$ \\
$n^{2}+20 n+9$ & 91 & $\left(\frac{364}{\cdot}\right)$ & $\sim 1.6887$ \\
\hline
\end{tabular}

Acknowledgements Open access funding provided by Graz University of Technology. This work was supported by a Hertha Firnberg grant of the Austrian Science Fund (FWF) [T846-N35]. The author thanks Christian Elsholtz for drawing her attention to this problem, Olivier Ramaré for commenting her preceding work [11] and the referee for his/her suggestions which led to improvement of the exposition.

Open Access This article is distributed under the terms of the Creative Commons Attribution 4.0 International License (http://creativecommons.org/licenses/by/4.0/), which permits unrestricted use, distribution, and reproduction in any medium, provided you give appropriate credit to the original author(s) and the source, provide a link to the Creative Commons license, and indicate if changes were made.

\section{References}

1. Akhilesh, P., Ramaré, O.: Explicit averages of non-negative multiplicative functions: going beyond the main term. Colloq. Math. 147(2), 275-313 (2017)

2. Bonciocat, N.C., Cipu, M., Mignotte, M.: On D(-1)-quadruples. Publ. Math. 56(2), 279-304 (2012)

3. Cohen, H.: A Course in Computational Algebraic Number Theory. Graduate Texts in Mathematics, vol. 138. Springer, Berlin (1993)

4. Delmer, F.: Sur la somme de diviseurs $\sum_{k \leq x}\{d[f(k)]\}^{s}$, C. R. Acad. Sci. Paris Ser. A B 272, A849A852 (1971)

5. Dudek, A.: On the number of divisors of $n^{2}-1$. Bull. Aust. Math. Soc. 93(02), 194-198 (2016) 
6. Elsholtz, C., Filipin, A., Fujita, Y.: On diophantine quintuples and $D(-1)$-quadruples. Monatsh. Math. 175(2), 227-239 (2014)

7. Elsholtz, C., Tao, T.: Counting the number of solutions to the Erdős-Straus equation on unit fractions. J. Aust. Math. Soc. 94(1), 50-105 (2013)

8. Erdős, P.: On the sum $\sum_{k=1}^{x} d(f(k))$. J. Lond. Math. Soc. 27, 7-15 (1952)

9. Hooley, C.: On the representation of a number as the sum of a square and a product. Math. Z. 69, 211-227 (1958)

10. Hooley, C.: On the number of divisors of quadratic polynomials. Acta Math. 110, 97-114 (1963)

11. Lapkova, K.: Explicit upper bound for an average number of divisors of quadratic polynomials. Arch. Math. (Basel) 106(3), 247-256 (2016)

12. Lapkova, K.: On the average number of divisors of reducible quadratic polynomials. Preprint, 2016. arXiv: 1704.06453

13. McKee, J.: On the average number of divisors of quadratic polynomials. Math. Proc. Camb. Philos. Soc. 117, 389-392 (1995)

14. McKee, J.: A note on the number of divisors of quadratic polynomials. Sieve methods, exponential sums, and their applications in number theory (Cardiff, 1995). In: London Mathematical Society Lecture Note Series 237, pp. 275-281. Cambridge University Press, Cambridge (1997)

15. McKee, J.: The average number of divisors of an irreducible quadratic polynomial. Math. Proc. Camb. Philos. Soc. 126(1), 17-22 (1999)

16. Pomerance, C.: Remarks on the Pólya-Vinogradov inequality. Integers 11, 531-542 (2011)

17. Ramaré, O.: An explicit density estimate for Dirichlet L-series. Math. Comput. 85(297), 325-356 (2016)

18. The LMFDB Collaboration: The L-Functions and Modular Forms Database. http://www.lmfdb.org (2016) (Online). Accessed 11 Nov 2016

19. Trudgian, T.: Bounds on the number of diophantine quintuples. J. Number Theory 157, 233-249 (2015)

20. Scourfield, E.J.: The divisors of a quadratic polynomial. Proc. Glasg. Math. Assoc. 5, 8-20 (1961)

21. Scourfield, E.J.: Smooth Divisors of Polynomials. Number Theory and Polynomials. Cambridge University Press, Cambridge (2008)

22. Washington, L.C.: Introduction to Cyclotomic Fields. Graduate Texts in Mathematics, vol. 83, 2nd edn. Springer, New York (1997) 